\title{
Group Acceptance Sampling Plans for Lifetimes Following an Odds Exponential LogLogistic Distribution
}

\author{
Rosaiah Kanaparthi ${ }^{1}$, Gadde Srinivasa Rao ${ }^{2 *}$, Kruthiventi \\ Kalyani $^{3}$ and Devireddy Charana Udaya Sivakumar ${ }^{3}$ \\ ${ }^{1}$ Department of Statistics, Acharya Nagarjuna University, Guntur - 522 \\ 510, India. \\ ${ }^{2}$ Department of Statistics, The University of Dodoma, P.O.Box: 259, \\ Tanzania. \\ ${ }^{3}$ UGC BSR Fellows, Department of Statistics, Acharya Nagarjuna \\ University, Guntur - 522 510, India. \\ *Corresponding Author E_mail:gaddesrao@gmail.com
}

Received: $29^{\text {th }}$ January 2016/ Revised: $13^{\text {th }}$ March 2017 / Accepted: $20^{\text {th }}$ March 2017 CIAppStat-SL2016

\begin{abstract}
A group acceptance sampling plan is developed for odds exponential log logistic distribution (OELLD) based on truncated lifetime with a known shape parameter. At a specified level of consumer risk and at the predefined test termination time, we derived the minimum number of groups required for a given group size and the acceptance number using the two-point approach by satisfying the producer's and the consumer's risks at the specified quality levels, while the termination time and the number of testers are specified. The values of operating characteristic (OC) function according to various quality levels are derived and the minimum ratios of true average life to the specified life for given producer's risk are obtained. The results are illustrated with real lifetime data set.
\end{abstract}

Key words: Odds exponential log logistic distribution, truncated life test, group acceptance sampling, producer's and consumer's risks.

\section{Introduction}

\subsection{Introduction to the Research Problem}

The reputation of companies depends upon the high reliability of their products. These companies compete with each other on the basis of quality and reliability. Thus quality control techniques became one of the most important tools to differentiate between the competitive 
enterprises in a global business market. In order to control the quality of the purchased goods, two major alternatives are open to a buyer. First type is the complete inspection, in which each and every item in the lot is to be inspected/ tested. This is often impractical and uneconomical and also involves cost and time for inspection. Secondly, the partial inspection in which a sample of items are taken which are inspected and the whole lot is accepted or rejected depending on whether few or more defective items are found in the sample.

Acceptance sampling plan is an essential tool in the statistical quality control. In most of the statistical quality control experiment, it is not possible to have a100\% inspection due to various reasons. In usual sampling plan, the decision of accepting or rejecting a lot is on the basis of a sample of items.

Acceptance sampling plans based on truncated life tests having single item as group were discussed for various distributions by Epstein (1954), Sobel and Tischendrof (1959), Goode and Kao (1961), Gupta (1962), Gupta and Groll (1961), Fertig and Mann (1980), Kantam and Rosaiah (1998), Kantamet al. (2001), Baklizi (2003), Baklizi and EL Masri (2004), Tsai and Wu (2006), Balakrishnanet al.(2007),Al-Nassar and Al-Omari (2013) and the reference therein.

The ordinary acceptance sampling scheme is used when the experimenter has the facility to test only one item at a time. In single sampling plans the experimenter needs more time to inspect the products and also samples to be tested. On the other hand, group acceptance sampling plans (GASPs) are used when the tester has the facility to install more than one item at the same time in a single tester. By doing so, substantial testing time and cost can be reduced if the tester to accommodate a multi number of items are utilized for testing purpose. The other importance of GASPs is that it provides strict inspection before the product is sent for the consumer's use. As such GASPs perform better than ordinary plans in terms of reduction of the time, strict inspection, cost, energy, minimize the risks and labor. 
To save time and cost in the life test, it is very often to put a number of items in a tester. In this life test, a tester is called a $\operatorname{group}(g)$ and the number of items $(r)$ in each tester is called the group size. The acceptance sampling via the group life test is called the group acceptance sampling plan (GASP) which is also often implemented under a truncated life test. For such a type of test, the determination of the sample size $n=r g$ is equivalent to determine the number of groups. This type of testers is frequently used in the case of so-called sudden death testing that is discussed by Pascual and Meeker (1998) and Vlceket al. (2003). Recently, Jun et al. (2006) introduced this group concept into acceptance sampling plan and developed variable sampling plans for sudden death testing for the Weibull distribution.

Rao (2009a, 2009b) presented a group acceptance sampling plans for lifetimes following a generalized exponential distribution and Marshall-Olkin extended Lomax distribution. Aslam and Jun (2009a,2009b) proposed a group acceptance sampling plan for truncated life tests based on the Weibull inverse Rayleigh and loglogistic distributions. Balamurali and Jun (2009) proposed a repetitive group sampling procedure for variables inspection. Radhakrishnan and Alagirisamy (2011) constructed a group acceptance sampling plan using weighted binomial distribution and Rao and Rao (2016) developed a two-stage group acceptance sampling plan based on life tests for half logistic distribution.

In group acceptance sampling plan(GASP), the items in each group are tested independently and under identical environmental conditions. Moreover, all the testers run simultaneously. The experiment is stopped at a pre-specified time $t$. If $c$ is the acceptance number for this experiment, then a lot is accepted if the recorded number of failures in each group is less than $c$ during the experimental time $t$. It is further assumed that the experimental time and the number of items in each group are prefixed in advance. Since $n=r g$, determining $n$ is equivalent to determining $g$. 


\subsection{Research Problem}

The main purpose of this article is to develop the group acceptance sampling plans for the odds exponential log-logistic distribution (OELLD) introduced by Rosaiah et al. (2016). Gupta (1962) suggested that for a skewed distribution, the median represents a better quality parameter than the mean. On the other hand, for a symmetric distribution, mean is preferable to use as a quality parameter. Since OELLD is a skewed distribution, we prefer to use the percentile lifetime as the quality parameter and it will be denoted by $t_{q}$. The rest of this article is organized as follows. In Section 2, we describe concisely the OELLD distribution and group acceptance sampling for lifetime percentiles under a truncated life test is discussed. The description of the proposed methodology with real lifetime data is presented in Section3. Comparison of distributions in Section 4 and finally, conclusions are made in Section 5.

\section{The Odds Exponential Log Logistic Distribution(OELLD)}

The OELLD was introduced and studied its properties by Rosaiah et al. (2016). The probability density function (pdf) and cumulative distribution function (cdf) of OELLD are respectively given by

$$
\begin{aligned}
& f(\mathrm{t} ; \sigma, \lambda, \alpha)=\frac{\theta}{\lambda \sigma}\left(\frac{x}{\sigma}\right)^{\theta-1} e^{-\frac{1}{\lambda}\left(\frac{x}{\sigma}\right)^{\theta}} \text { for } t>0, \sigma, \lambda, \theta>0 \\
& \text { and } F(\mathrm{t} ; \sigma, \lambda, \alpha)=1-e^{-\frac{1}{\lambda}\left(\frac{x}{\sigma}\right)^{\theta}}, t>0, \lambda, \sigma>0 \text { and } \alpha>0 .
\end{aligned}
$$

where $\sigma, \lambda$ are the scale parameters and $\theta$ is shape parameter. This OELLD distribution has increasing failure rate (IFR) for the shape parameter $\theta>1$, decreasing failure rate (DFR) for $\theta<1$. This shows that this distribution can be used for industrial applications and reliability studies.

The $100 q$-th percentile of the OELLD is given by

$$
t_{q}=\sigma \eta_{q}, \quad \text { where } \quad \eta_{q}=[-\lambda \ln (1-q)]^{1 / \theta}
$$


Hence, for the fixed values of $\lambda=\lambda_{0}$ and $\theta=\theta_{0}$, the quantile $t_{q}$ given in Equation (3) is the function of scale parameter $\sigma=\sigma_{0}$, that is $t_{q} \geq t_{q}^{0} \Leftrightarrow \sigma \geq \sigma_{0}$, where $\sigma_{0}=t_{q}^{0}\left[-\lambda_{0} \ln (1-q)\right]^{-1 / \theta_{0}}$

Note that $\sigma_{0}$ depends on $\lambda_{0}$ and $\theta_{0}$, to build up acceptance sampling plans for the OELLD, ascertain $t_{q} \geq t_{q}^{0}$, equivalently that $\sigma$ exceeds $\sigma_{0}$.

\subsection{The Group Acceptance Sampling Plan (GASP)}

Here, we discussed group acceptance sampling plans (GASPs) when lifetime of the product follows OELLD. We considered the GASP under the truncated life test, which is based on the total number of failures from all groups. The procedure for the proposed plan is as follows [Aslam et al. (2011)]:

- Randomly draw a sample of size $n$ from a production lot, allocate $r$ items to each of $g$ groups (or testers) so that $n=r g$ and put them on test until the pre-determined test time $t_{0}$.

- Accept the lot when the number of failures from each $g$ groups is smaller than or equal to $c$. Truncate the test and reject the lot as soon as the number of failures from all $g$ groups is larger than $c$ before $t_{0}$.

The probability of accepting a lot for the group acceptance sampling plan $\mathrm{P}(p)$ based on the number of failures from all groups under a truncated life test at the test time schedule $t_{0}$ is

$$
\mathrm{P}(p)=\sum_{i=0}^{c}\left(\begin{array}{l}
r g \\
i
\end{array}\right) p^{i}(1-p)^{r g-i}
$$

Where $g$ is the number of testers, $c$ is the acceptance number, $r$ is the group size and $p$ is the probability of getting a failure within the life test time schedule, $t_{0}$. If the product lifetime follows OELLD, then $p=F\left(t_{0} ; \sigma_{0}, \lambda_{0}, \alpha_{0}\right)$. Usually, it would be convenient to determine the 
experiment termination time, $t_{0}$, as $t_{0}=\delta_{q}^{0} t_{q}^{0}$ for a constant $\delta_{q}^{0}$ and the targeted $100 \mathrm{q}$-th lifetime percentile, $t_{q}^{0}$. Let $t_{q}$ be the true $100 \mathrm{q}$-th lifetime percentile. Then, $\mathrm{p}$ can be rewritten as

$$
p=1-\exp \left\{-\frac{1}{\lambda}\left(\frac{t_{0}}{\sigma}\right)^{\theta}\right\}=1-\exp \left\{-\frac{1}{\lambda}\left(\frac{\eta_{q} \delta_{q}^{\mathrm{o}}}{\left(t_{q} / t_{q}^{\mathrm{o}}\right)}\right)^{\theta}\right\}
$$

The design parameters of the proposed GASP are obtain using two points approach on the OC curve by considering the producer's and consumer's risks. In this method the quality level is studied with the ratio of its quantile to the lifetime, $t_{q} / t_{q}^{0}$. The producer's point of view is the probability of lot acceptance should be at least $1-\alpha$ at the acceptable reliability level (ARL), $p_{1}$. So the producer demands that a lot should be accepted at various levels, say $t_{q} / t_{q}^{0}=2,4,6,8$ in Equation (6). Whereas, the consumer's viewpoint is the lot rejection probability should be at most $\beta$ at the lot tolerance reliability level (LTRL), $p_{2}$. In this way, the consumer considers that a lot should be rejected when $t_{q} / t_{q}^{0}=1$, in Equation (6). Now, let us consider

$$
\begin{aligned}
& P_{a}\left(p_{1}\right)=\sum_{i=0}^{c}\left(\begin{array}{l}
r g \\
i
\end{array}\right) p_{1}^{i}\left(1-p_{1}\right)^{r g-i} \geq 1-\alpha(7) \\
& P_{a}\left(p_{2}\right)=\sum_{i=0}^{c}\left(\begin{array}{l}
r g \\
i
\end{array}\right) p_{2}^{i}\left(1-p_{2}\right)^{r g-i} \leq \beta(8)
\end{aligned}
$$

where $p_{1}$ and $p_{2}$ are

$$
p_{1}=1-\exp \left\{-\frac{1}{\lambda}\left(\frac{\eta_{q} \delta_{q}^{0}}{\left(t_{q} / t_{q}^{0}\right.}\right)^{\theta}\right) \text { and } \mathrm{p}_{2}=1-\exp \left\{-\frac{1}{\lambda}\left(\eta_{q} \delta_{q}^{0}\right)^{\theta}\right\}
$$

For a specified producer's risk $\alpha=0.05$ and termination time schedule $t_{0}=\delta_{q} t_{q}^{0}$ with $\delta_{q}^{0}=0.5$ or 1.0 , the two parameters of the proposed group acceptance sampling plan under the truncated life test at the prespecified time, $t_{0}$, with $\lambda=2$ and $\theta=1,1.5,2$ are obtained according 
Group Acceptance Sampling Plans for Lifetimes Following an Odds Exponential Log Logistic Distribution

to the consumer's confidence levels $\beta=0.25,0.10,0.05,0.01$ for $50^{\text {th }}$ percentile and presented in Tables 1, 2and 3 .

The maximum likelihood estimates (MLEs) of the parameters $\lambda$ and $\theta$ are obtained (i.e., $\hat{\lambda}=20.4032$ and $\hat{\theta}=2.7932$ ) from the lifetime data set given in the following Section 3.The plan parameters are determined using the MLEs $\hat{\lambda}=20.4032$ and $\hat{\theta}=2.7932$ at $50^{\text {th }}$ percentile and are presented in Table 4 . The operating characteristic values are also calculated for these values. From Tables 1 to 4 , we observe that the percentile ratio increases when the number of groups $g$ reduces. Similarly, as $r$ increases from 5 to 10, the number of groups reduces.

\section{Description of the Proposed Methodology with Real Lifetime Data}

The proposed methodology is illustrated with following real lifetime data on breaking stress of carbon fibers (in Gba) and it is taken from Nichols and Padgett (2006).

$0.39,0.81,0.85,0.98,1.08,1.12,1.17,1.18,1.22,1.25,1.36,1.41,1.47,1.57,1$ $.57,1.59,1.59,1.61,1.61,1.69,1.69,1.71,1.73,1.80,1.84,1.84,1.87,1.89,1$. $92,2.00,2.03,2.03,2.05,2.12,2.17,2.17,2.17,2.35,2.38,2.41,2.43,2.48,2.4$ $8,2.50,2.53,2.55,2.55,2.56,2.59,2.67,2.73,2.74,2.76,2.77,2.79,2.81,2.81$ ,2.82,2.83,2.85,2.87,2.88,2.93,2.95,2.96,2.97,2.97,3.09,3.11,3.11,3.15, $3.15,3.19,3.19,3.22,3.22,3.27,3.28,3.31,3.31,3.33,3.39,3.39,3.51,3.56,3$ $.60,3.65,3.68,3.68,3.68,3.70,3.75,4.20,4.38,4.42,4.70,4.90,4.91,5.08,5$. 56.

We fitted the OELLD curve for the above data which is shown in the Figure 1: 

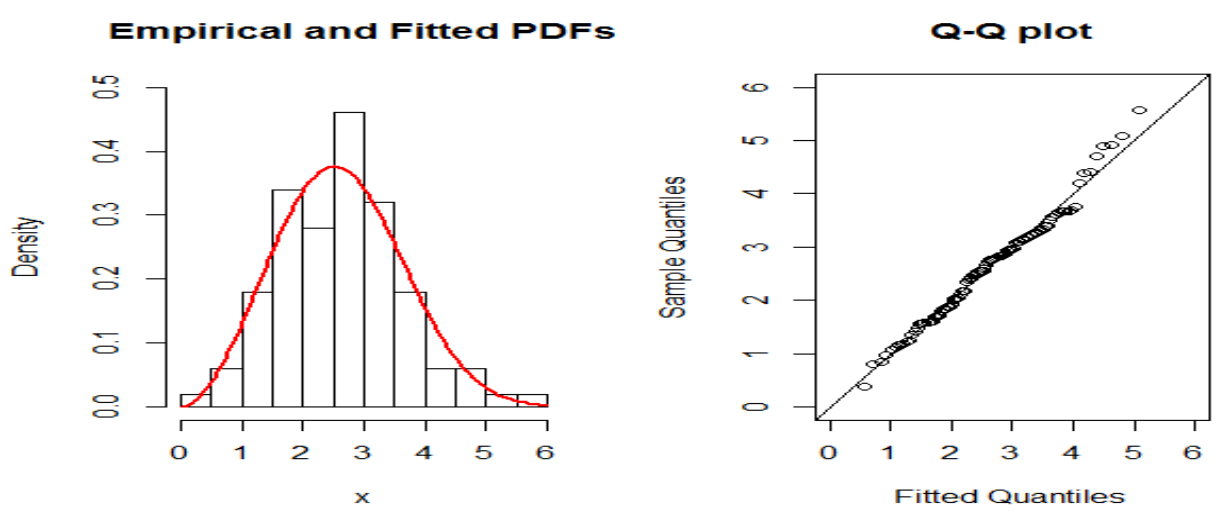

Figure 1. Estimated density and Q-Q plot for OELLD.

The parameters of OELLD for breaking stress of carbon fibers are obtained using maximum likelihood estimation and are given by $\hat{\lambda}=20.4032$ and $\hat{\theta}=2.7932$. The goodness of fit is tested using the Kolmogorov-Smirnov test, we found that the distance between the data and the fitted OELLD is 0.0604 with p-value is 0.8582 . Hence, the two-parameter OELLD provides reasonable fit for the breaking stress of carbon fibers and Figure 1 also emphasized the same.

Since the life times of a product given in the above data follows OELLD with MLEs $\hat{\lambda}=20.4032$ and $\hat{\theta}=2.7932$. Suppose that it desired to develop the group acceptance sampling plan to satisfy that the $50^{\text {th }}$ percentile lifetime is greater than breaking stress of carbon fibers 0.40 through the experiment to be completed breaking stress of carbon fibers by 0.80 to protect the producer's risk at $5 \%$.For $\hat{\lambda}=20.4032$ and $\hat{\theta}=2.7932$, the consumer's risk is $25 \%, r=5$, $\delta_{q}^{0}=1.0$ and $t_{q} / t_{q}^{0}=2$, the minimum number of groups and acceptance number given by $g=2$ and $c=3$ from Table 4 . Thus the design can be implemented as follows: select a total of 10 products, and allocate five items to each of the two groups. We can accept the lot when no more than three failures occur before breaking stress of carbon fibers 0.80 from each of two groups. According to this plan, the breaking stress of 
carbon fibers could have been accepted because there is only one failure before the termination time 0.80 .

\section{Comparison of Distributions}

In the Table 5 we compare the plan parameters for the generalized log logistic distribution (GLLD) studied by Aslam et al. (2011), Type-II generalized log logistic distribution (TGLLD) developed by Rosaiah et al. (2016) and OELLD when $\lambda=2, \theta=2, \beta=0.10$ and $r=5, \delta_{q}^{0}=0.5$. The acceptance number for the OELLD is smaller as compared to generalized log-logistic distribution by using to $50^{\text {th }}$ percentile.

\section{Conclusions}

In this article, we studied a group acceptance sampling plans when lifetime of the product follows OELLD. The plan parametric quantities like the number of groups, $g$, and acceptance number, care determined by considering the consumer's risk and producer's risk simultaneously. Our proposed plan noticed that if the percentile ratio increases, the number of groups $g$ reduces and as $r$ increases the number of groups reduces for all the parametric combinations considered in this article. The plan also illustrated with real lifetime data for industrial purpose.

\section{Acknowledgements:}

The authors are deeply thankful to the editor and reviewers for their valuable suggestions to improve the manuscript. The third and fourth authors are extended their thanks to the University Grants Commission, New Delhi, India for sanctioning BSR fellowships to do the research work. 


\section{References}

1. Al-Nassar, A.D. and Al-Omari, A.I. (2013). Acceptance sampling plan based on truncated life tests for exponentiated Fréchet distribution.Journal of Statistics and Management Systems, 16(1):13-24. http://dx.doi.Org/10.1080/09720510.2013.777571.

2. Aslam, M. and Jun, C.H. (2009a). A group acceptance sampling plan for truncated life test having Weibull distribution. Journal of Applied Statistics, 36:1021-1027. DOI: 10.1080/02664760802566788.

3. Aslam, M., and Jun, C.H. (2009b). A group acceptance sampling plans for truncated life tests based on the inverse Rayleigh and log-logistic distributions. Pakistan Journal of Statistics, 25(2): 107-119.

4. Aslam, M. Jun, C.H. and Ahmad, M. (2009). A group sampling plan based on truncated life test for gamma distributed items.Pakistan Journal of Statistics, 25(3): 333-340.

5. Aslam, M., Kundu, D., Jun, C.H., and Ahmad, M. (2011). Time Truncated Group Acceptance Sampling Plans for generalized exponential distribution. Journal of Testing and Evaluation, 39(4): 671-677.

6. Aslam, M., Shaib, M., Jun, C-H and Nadia, S. (2011). Time truncated group acceptance sampling plans for lifetime percentiles under generalized log-logistic distributions. International Journal of Current Research and Review, 3(11): 23-35.

7. Baklizi, A. (2003). Acceptance sampling based on truncated life tests in the Pareto distribution of the second kind. Advances and Applications in Statistics, 3:33-48.

8. Baklizi, A. and EI Masri, A.E.K. (2004). Acceptance sampling based on truncated life tests in the Birnbaum-Saunders model.Risk Analysis,24: 1453. http://dx.doi.org/10.1111/j.02724332.2004.00541.x

9. Balamurali, S. and Jun, C.H. (2009). Repetitive group sampling procedure for variables inspection. Journal of Applied Statistics, 33(10):

1159-1172.

http://dx.doi.org/10.1080/02664760500446010. 
Group Acceptance Sampling Plans for Lifetimes Following an Odds Exponential Log Logistic Distribution

10. Balakrishnan, N., Leiva, V. and Lopez, J. (2007). Acceptance sampling plans from truncated life tests based on the generalized Birnbaum-Saunders distribution. Communication in StatisticsSimulation and Computation, 36:643-656. DOI: 10.1080/03610910701207819.

11. Epstein, B. (1954). Truncated life tests in the exponential case.Annals of Mathematical Statistics, 25:555-564.

12. Fertig, F.W. and Mann, N.R. (1980). Life-test sampling plans for two-parameter Weibull populations. Technometrics, 22, 165-177. DOI: $10.2307 / 1268455$

13. Goode, H.P. and Kao, J.H.K. (1961). Sampling plans based on the Weibull distribution. Proceeding of the Seventh National Symposium on Reliability and Quality Control, pp. 24-40, Philadelphia.

14. Gupta, S.S. (1962). Life test sampling plans for normal and lognormal distribution. Technometrics, 4:151- 175. DOI: $10.2307 / 1266616$

15. Gupta, S.S. and Groll, P.A. (1961). Gamma distribution in acceptance sampling based on life tests. J. Amer. Statist. Assoc., 56: 942-970. DOI: 10.2307/2282006.

16. Jun, C.H., Balamurali, S. and Lee, S.H. (2006). Variables sampling plans for Weibull distributed lifetimes under sudden death testing. IEEE Transactions on Reliability, 55: 53-58. DOI: 10.1109/TR.2005.863802.

17. Kantam, R.R. L. and Rosaiah, K. (1998). Half-logistic distribution in acceptance sampling based on life tests.IAPQR Transactions, 23(2): 117-125.

18. Kantam, R.R. L., Rosaiah, K. and Rao, G.S. (2001). Acceptance sampling based on life tests: Log-logistic model.Journal of Applied Statistics, 28:121-128. http://dx.doi.org/10.1080/02664760120011644.

19. Lemonte, A.J. (2014). The beta log-logistic distribution. Brazilian Journal of Probability and Statistics, 28(3): 313-332. DOI: 10.1214/14-BJPS260 
20. Nichols, M. D. and Padgett, W. J. (2006). A Bootstrap control chart for Weibull percentiles. Quality and Reliability Engineering International, 22: 141-151. DOI: 10.1002/qre.691

21. Pascual, F.G. and Meeker, W.Q. (1998). The modified sudden death test: Planning life tests with a limited number of test positions. Journal of Testing and Evaluation, 26: 434-443.

22. Radhakrishnan R. and Alagirisamy K. (2011). Construction of group acceptance sampling plan using weighted binomial distribution. International Journal of Recent Scientific Research, 2(7): 229-231.

23. Rao G.S (2009a). A group acceptance sampling plans for lifetimes following a generalized exponential distribution. Economic Quality Control, 24 (1): 75-85. DOI: 10.1515/EQC.2009.75.

24. Rao, G.S. (2009b). A group acceptance sampling plans on truncated life tests for Marshall-Olkin extended Lomax distribution. Electron. J. App. Stat. Anal. 3 (1):18-27. DOI 10.1285/i20705948v3n1p18.

25. Rao, B.S. and Rao, G.S. (2016).A two-stage group acceptance sampling plan based on life tests for half logistic distribution, Model Assisted Statistics and Applications, 11(3): 203-211.

26. Rosaiah, K., Rao, G.S., and Prasad, S.V.S.V.S.V. (2016). A group acceptance sampling plans based on truncated life tests for Type-II generalized log logistic distribution, ProbStat Forum, 9, 88-94.

27. Rosaiah, K., Rao, G.S., Kalyani, K. and Sivakumar, D.C.U. (2016). Odds exponential log logistic distribution: properties and estimation. To appear in Journal of mathematics and Statistics.DOI: 10.3844/jmssp.2017

28. Sobel, M. and Tischendrof, J. A. (1959). Acceptance sampling with new life test Objectives. Proceedings of Fifth National Symposium on Reliability and Quality Control, Philadelphia, Pennsylvania, pp.108-118.

29. Tsai, T.R. and Wu, S.J. (2006). Acceptance sampling based on truncated life tests for generalized Rayleigh distribution.Journal of Applied Statistics, 33: $595-$ 600.http://dx.doi.org/10.1080/02664760600679700. 
Group Acceptance Sampling Plans for Lifetimes Following an Odds Exponential Log Logistic Distribution

30. Vlcek, B.L., Hendricks, R.C. and Zaretsky, E.V. (2003). Monte Carlo Simulation of Sudden Death Bearing Testing. NASA, Hanover, MD, USA.

Table 1:GASPfor OELLD with $\lambda=2$ and $\theta=1$ for $50^{\text {th }}$ percentile

\begin{tabular}{|c|c|c|c|c|c|c|c|c|c|c|c|}
\hline \multirow{3}{*}{$\beta$} & \multirow{3}{*}{$t_{q} / t_{q}^{0}$} & \multicolumn{6}{|c|}{$r=5$} & \multicolumn{4}{|c|}{$\mathrm{r}=10$} \\
\hline & & \multirow{2}{*}{\multicolumn{3}{|c|}{$\begin{array}{c}\delta_{q}^{0}=0.5 \\
P_{a}\left(p_{1}\right)\end{array}$}} & \multirow{2}{*}{\multicolumn{3}{|c|}{$\begin{array}{r}\delta_{q}^{0}=1.0 \\
P_{a}\left(p_{1}\right)\end{array}$}} & \multicolumn{2}{|r|}{$\delta_{q}^{0}=0.5$} & \multicolumn{2}{|c|}{$\delta_{q}^{0}=1.0$} \\
\hline & & & & & & & & & $P_{a}\left(p_{1}\right)$ & c & $P_{a}\left(p_{1}\right)$ \\
\hline 25 & & & & 0.9540 & & & -- & & -- & - & \\
\hline & 4 & 5 & 5 & 54 & 5 & 3 & 0.97795 & 3 & 0.96576 & 2 & 0.9705 \\
\hline & 6 & 2 & 3 & & 3 & 2 & & & 0.9 & & .982 \\
\hline & 8 & 2 & 3 & 4 & 2 & 2 & 0.9 & 2 & 0.9 & 1 & 0.956 \\
\hline .10 & & -- & -- & . & -- & -- & -- & -- & -- & -- & -- \\
\hline & 4 & 5 & 6 & 0.96 & 6 & 4 & $0.9705 \mid 5$ & 3 & 0.96576 & 2 & 0.970 \\
\hline & 6 & 3 & 5 & 2 & 4 & 3 & 4 & 3 & 0.9 & 2 & 83 \\
\hline & 8 & 3 & 5 & 0 & 2 & 2 & 0.95603 & 3 & 0.96334 & 2 & 0.978 \\
\hline .05 & & -- & -- & - & -- & -- & -- & -- & -- & -- & -- \\
\hline & 4 & 6 & 8 & 4 & 7 & 5 & 0.96506 & 4 & 0.9 & 3 & 0.961 \\
\hline & 6 & 3 & 5 & 2 & 5 & 4 & 3314 & 3 & 0.97555 & 2 & .983 \\
\hline & 8 & 3 & 5 & 0.9800 & 3 & 3 & 0.96923 & 3 & 0.96334 & 2 & 0.978 \\
\hline 0.01 & & -- & -- & -- & -- & -- & - & -- & -- & -- & - \\
\hline & 4 & 8 & 11 & 7 & 8 & 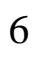 & 9 & 6 & 0.9 & & 0.9 \\
\hline & 6 & 5 & 9 & & 5 & J & & & & & \\
\hline & 8 & 4 & 0 & 38 & 4 & 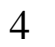 & & & 0.9 & & 0.978 \\
\hline
\end{tabular}


Table 2: GASP for OELLD with $\lambda=2$ and $\theta=1.5$ for $\mathbf{5 0}^{\text {th }}$ percentile.

\begin{tabular}{|c|c|c|c|c|c|c|c|c|c|c|c|c|c|}
\hline \multirow{3}{*}{$\beta$} & \multicolumn{7}{|c|}{$r=5$} & \multicolumn{6}{|c|}{$r=10$} \\
\hline & \multirow[t]{2}{*}{$t_{q} / t_{q}^{0}$} & \multicolumn{3}{|c|}{$\delta_{q}^{0}=0.5$} & \multicolumn{3}{|c|}{$\delta_{q}^{0}=1.0$} & \multicolumn{3}{|c|}{$\delta_{q}^{0}=0.5$} & \multicolumn{3}{|c|}{$\delta_{q}^{0}=1.0$} \\
\hline & & c & $\mathrm{g}$ & $P_{a}\left(p_{1}\right)$ & c & $\mathrm{g}$ & $P_{a}\left(p_{1}\right)$ & c & $\mathrm{g}$ & $P_{a}\left(p_{1}\right)$ & c & $\mathrm{g}$ & $P_{a}\left(p_{1}\right)$ \\
\hline 25 & & 6 & 8 & 0.95549 & & 5 & 0.9699 & 6 & 4 & 0.9554 & 10 & 3 & 0.9552 \\
\hline & 4 & 2 & 4 & 0.9 & & 2 & 0.95 & 2 & 2 & & 2 & 1 & \\
\hline & 6 & 1 & 3 & & & 1 & & 1 & 2 & & 2 & 1 & \\
\hline & 8 & 1 & 3 & & & 1 & & 1 & 2 & & 1 & 1 & \\
\hline .10 & & 9 & 13 & 0. & & 6 & & 10 & 7 & & 10 & 3 & 0 . \\
\hline & 4 & 2 & 5 & 0.9 & & 2 & 0.9 & 3 & 3 & & 2 & 1 & 0. \\
\hline & 6 & 1 & 4 & & & 2 & 0. & 1 & 2 & & 2 & 1 & \\
\hline & 8 & 1 & 4 & & & 2 & & 1 & 2 & & 1 & 1 & 0.9 \\
\hline .05 & & 10 & 15 & & & 6 & & 11 & 8 & & 10 & 3 & 0. \\
\hline & 4 & 3 & 7 & & & 3 & 0.9 & 3 & 4 & & $\mathbf{4}$ & 2 & 0.9 \\
\hline & 6 & 1 & 14 & & & 3 & & 1 & 2 & & 3 & 2 & \\
\hline & 8 & 1 & 4 & 0.9 & & 2 & & 1 & 2 & 0.9806 & 1 & 1 & 0.965 \\
\hline 01 & & -- & -- & -- & 14 & 9 & 0.9 & -- & -- & -- & - & -- & -- \\
\hline & 4 & 3 & 9 & & & 4 & & 4 & 5 & & 4 & 2 & 0.9 \\
\hline & 6 & 2 & 8 & & & 3 & $0.9^{\prime}$ & 2 & 4 & & 3 & 2 & 0.987 \\
\hline & o & 1 & 0 & 0.95862 & & 5 & 0.9905 & 1 & 3 & 0.9586 & 2 & 2 & 0.978 \\
\hline
\end{tabular}


Table 3: GASP forOELLD with $\lambda=2$ and $\theta=2$ for $\mathbf{5 0}^{\text {th }}$

\begin{tabular}{|c|c|c|c|c|c|c|c|c|c|c|c|c|c|}
\hline & \multicolumn{13}{|c|}{ percentile } \\
\hline \multirow{3}{*}{$\beta$} & \multirow{3}{*}{$t_{q} / t_{q}^{0}$} & 5 & & & & & & & & & & & \\
\hline & & \multicolumn{3}{|c|}{$\delta_{q}^{0}=0.5$} & \multicolumn{3}{|c|}{$\delta_{q}^{0}=1.0$} & \multicolumn{3}{|c|}{$\delta_{q}^{0}=0.5$} & \multicolumn{3}{|c|}{$\delta_{q}^{0}=1.0$} \\
\hline & & c & $\mathrm{g}$ & $P_{a}\left(p_{1}\right)$ & c & $\mathrm{g}$ & $P_{a}\left(p_{1}\right)$ & c & $\mathrm{g}$ & $P_{a}\left(p_{1}\right)$ & 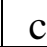 & $\mathrm{g}$ & $P_{a}\left(p_{1}\right)$ \\
\hline \multirow[t]{4}{*}{0.25} & & 4 & 8 & 0.9738 & & 3 & 0.97 & 4 & 4 & 0.9738 & 6 & 2 & 0.970 \\
\hline & 4 & 1 & 4 & 0.9806 & & 1 & 0.98 & 1 & 2 & 0.9806 & 2 & 1 & 0.9927 \\
\hline & 6 & 0 & 2 & 0.95 & & 1 & & 0 & 1 & & 1 & 1 & \\
\hline & 8 & 0 & 2 & 0.9 & & 1 & & 0 & 1 & & 1 & 1 & \\
\hline \multirow[t]{4}{*}{0.10} & & 5 & 12 & 0.9 & & 4 & & 5 & 6 & & 6 & 2 & 0.9705 \\
\hline & 4 & 1 & 5 & 0.97 & & 2 & 0.9 & 1 & 3 & & 2 & 1 & 0.5 \\
\hline & 6 & 1 & 5 & 0.9 & & 2 & & 1 & 3 & & 1 & 1 & \\
\hline & 8 & 0 & 3 & 0.9 & & 2 & & 1 & 3 & & 1 & 1 & \\
\hline \multirow[t]{4}{*}{0.05} & & 6 & 15 & 0.966 & & 5 & 0.9650 & 7 & 8 & & 8 & 3 & 0.9610 \\
\hline & 4 & 1 & 6 & 0.95 & & 3 & 0.9 & 1 & 3 & 0.9 & 3 & 2 & 0.9909 \\
\hline & 6 & 1 & 6 & 0.9 & & 2 & & 1 & 3 & 98 & 1 & 1 & .9852 \\
\hline & 8 & 1 & 6 & 0.99 & & 2 & 0.9 & 1 & 3 & & 1 & 1 & .9951 \\
\hline \multirow{4}{*}{0.01} & & 8 & 21 & 0.9 & & 6 & & 8 & 11 & & 8 & 3 & 0.9610 \\
\hline & 4 & 2 & 10 & 0.98 & & 3 & 4 & 2 & 5 & 0.9 & 3 & 2 & 0.9909 \\
\hline & 6 & 1 & 8 & 0.98 & & 3 & 0.9676 & 1 & 4 & 0.9 & 2 & 2 & 0.9938 \\
\hline & 8 & 1 & 8 & 0.9947 & & 3 & 0.9889 & 1 & 4 & 0.9947 & 1 & 2 & 0.9806 \\
\hline
\end{tabular}


Table 4:GASP forOELLD with $\hat{\lambda}=20.4032$ and $\hat{\theta}=2.7932$ for $\mathbf{5 0}^{\text {th }}$ percentile.

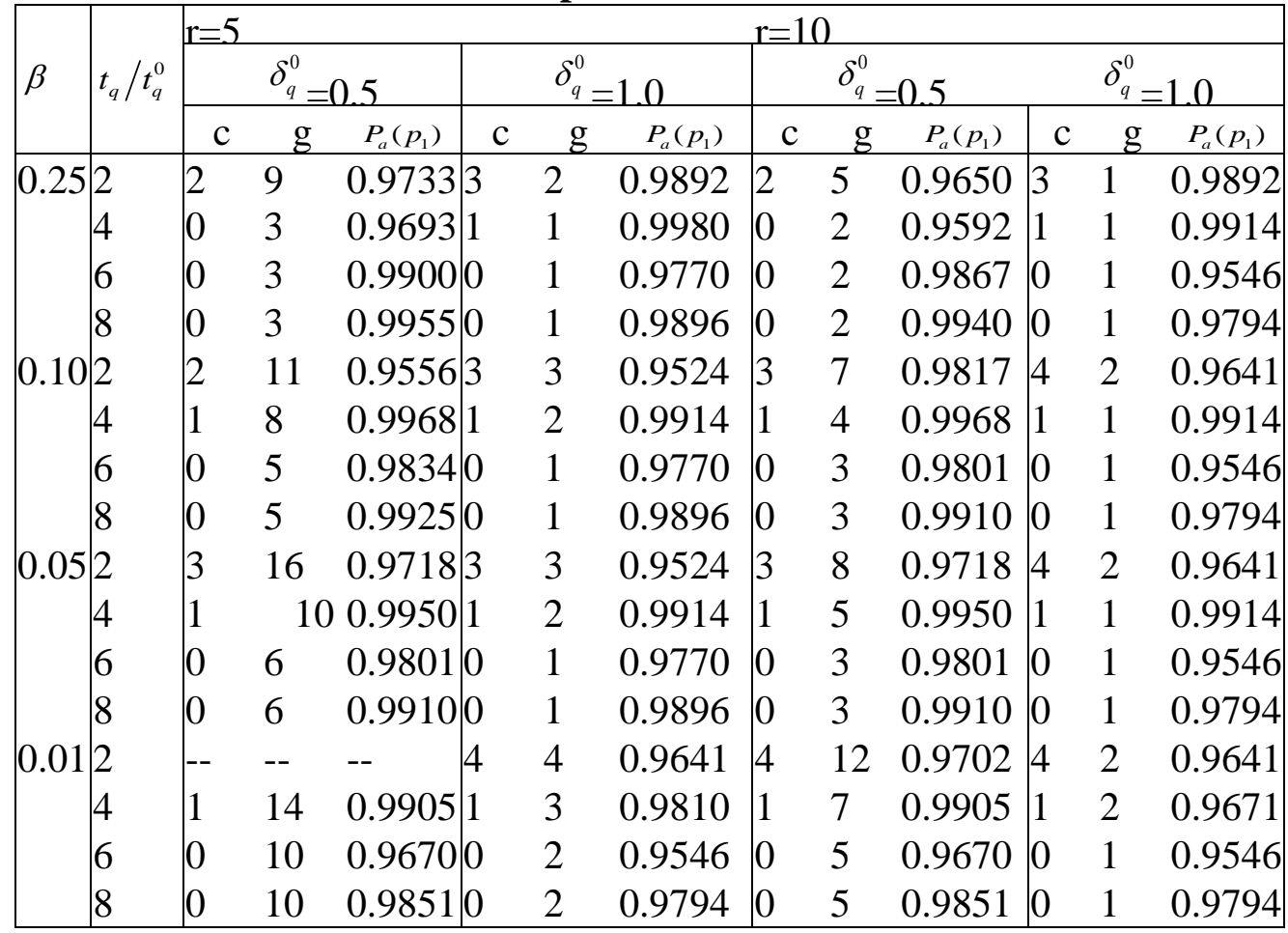

Table 5.Comparison between generalized log-logistic distribution and OELLD

\begin{tabular}{|c|c|c|c|c|c|c|c|c|c|}
\hline \multirow[b]{2}{*}{$t_{q} / t_{q}^{0}$} & \multicolumn{3}{|c|}{ GLLD } & \multicolumn{3}{|c|}{ OELLD } & \multicolumn{3}{|c|}{ TGLLD } \\
\hline & $\mathrm{c}$ & $\mathrm{g}$ & $P P_{a}\left(p_{1}\right)$ & $\mathrm{c}$ & $\mathrm{g}$ & $P_{a}\left(p_{1}\right)$ & $\mathrm{c}$ & g & $\overline{P_{a}\left(p_{1}\right)}$ \\
\hline 2 & 1 & 69 & \begin{tabular}{l|l|}
0.99595 \\
\end{tabular} & & 12 & 0.9587 & 8 & 4 & 0.9637 \\
\hline 4 & 0 & 41 & 0.99901 & & 5 & 0.9705 & 1 & 1 & 0.9559 \\
\hline 6 & 0 & 41 & 0.9999 1 & & 5 & 0.9936 & 1 & 1 & 0.9900 \\
\hline 8 & 0 & & 0.99990 & & 3 & 0.9602 & 1 & 1 & 0.9967 \\
\hline
\end{tabular}

